\title{
Pistacia terebinthus extract: As a potential antioxidant, antimicrobial and possible $\beta$-glucuronidase inhibitor
}

\author{
Dilek Demirbüker Kavak, Evren Altık*, Oğuz Bayraktar, Semra Ülkü \\ Izmir Institute of Technology, Chemical Engineering Department, Gülbahçe Köyü 35430, Urla Izmir, Turkey
}

\section{A R T I C L E I N F O}

Article history:

Available online 4 February 2010

\section{Keywords:}

B-Glucuronidase

Pistacia terebinthus

Plant extract

Inhibition kinetics

\begin{abstract}
A B S T R A C T
In this study, inhibition of Escherichia coli glucuronidase (GUS) by a plant extract of Pistacia terebinthus was investigated. P. terebinthus (from Karaburun region in Turkey) leaf extract exhibits various bioactivities, such as antioxidant, antimicrobial and cytotoxic property because of its flavonoid, phenolic and alkaloid contents. HPLC analyses were performed to evaluate the composition of the extract and main flavonoid compounds such as luteolin, luteolin-7-glucoside and apigenin-7-glucoside were detected in extract. GUS activity and kinetic data of the GUS were obtained by measuring rate of production of resulting fluorescent 4-methylumbelliferone (4-MU) and phenolphthalein fluorometrically and spectrophotometrically, respectively. Plant leaves showed higher antioxidant capacity as 85 TEAC (trolox equivalent antioxidant capacity) value and higher antimicrobial activity towards Staphylococcus aureus. 92.4\% GUS enzyme inhibition was achieved with $8.3 \mu \mathrm{g} \mathrm{ml}^{-1}$ crude extract concentration, where $\mathrm{IC}_{50}$ value was found as $2.1( \pm 0.2) \mu \mathrm{g} \mathrm{ml}^{-1}$. Kinetic studies by nonlinear regression revealed that inhibition type caused by plant extract was mixed type (noncompetitive and competitive together). $K_{\mathrm{m}}$ and $V_{\max }$ values were $96.94 \pm 9.68 \mathrm{nM}$ and $7.81 \pm 0.16 \mathrm{nmol} \mathrm{min}^{-1} \mathrm{mg}^{-1}$, respectively. Inhibition constant $\left(\alpha K_{i}\right)$ was found as $7.00 \pm 0.93 \mathrm{nM}$ having $\alpha$ value $2.17 \pm 0.93$. Our results may provide useful information regarding the inhibition of microbial GUS activity, preventing deglucuronidation and reducing possible cancer risk by bioactive P. terebinthus crude extract.
\end{abstract}

(C) 2010 Elsevier B.V. All rights reserved.

\section{Introduction}

Glucuronidation is the process of chemically binding a substance to glucuronic acid via a glycosidic bond. By this process glucuronide conjugates are formed and this process can be considered as a detoxification mechanism. As a result of glucuronidation those conjugates are much more water soluble than the original substance. Also because of the high molecular weight and polar nature, they can be easily excreted via the bile into the small intestine.

Glucuronidation is used to excrete different xenobiotics such as drugs or pollutants and also to excrete bilirubin, androgens, estrogens, mineralocorticoids, glucocorticoids, fatty acid derivatives, retinoids and bile acids. Most of this process is done by liver. These exogenous compounds are poorly reabsorbed into the bloodstream and are efficiently eliminated from the body unless they are hydrolyzed [1]. $\beta$-Glucuronidase (GUS) enzyme is of interest that hydrolyses those biliary glucuronides. It is found in some mammalian and plant tissues. In intestine, its activity is mostly bacterial

\footnotetext{
* Corresponding author at: Izmir Institute of Technology, Department of Chemical Engineering, Biochemical Engineering Research Laboratory (BERL), Gulbahce Koyu 35430, Urla-Izmir, Turkey. Tel.: +90 232 7506674; fax: +90 2327506645.

E-mail address: evrenaltiok@iyte.edu.tr (E. Altık).
}

origin and it has been detected among bacterial genera belonging to the dominant human intestinal microbiota. Many bacteria, such as Escherichia coli, Klebsiella sp., Clostridium sp., Bacteroides fragilis, Streptococcus sp., Staphylococcus sp., Bacillus sp., Enterococcus sp. and Corynebacterium sp. also were reported to possess GUS activity $[2,3]$. Some of the glucuronidase that are secreted through the biliary route into the intestine may be hydrolyzed by the action of those bacterial GUS present in the intestine [4] and their hydrolysis may liberate free toxins. This process is very important since it increases endogenous exposure of colon to carcinogens. After hydrolysis by bacterial GUS, glucuronidation process is reversed and more toxic parent compound is not excreted but absorbed from the intestine entering the enterohepatic circulation. If glucuronide hydrolysis is a rate limiting step in this process, then the activity of microbial $\beta$-glucuronidase in the colon may influence the risk of colon carcinogenesis [5]. Rafter et al. [6] reported that the activity of $\beta$-glucuronidase in the colon can alter tumour induction in animal models of colorectal cancer. The use of a $\beta$-glucuronidase inhibitor with conjugate carcinogen azoxymethane significantly reduced the number of tumours formed in the rat colon. This result indicated important role of microbial $\beta$-glucuronidase in tumour induction. Results of different studies suggest that GUS activity can be considered as a cancer risk biomarker and its activity is related to cancer development and number of carcinogens 
being produced by hydrolysis of glucuronides in the intestine [7].

Certain plants with their high antioxidant potential are widely used in phytopharmacy and take a role in reducing incidence of cancer. Besides, recent studies showed that also some of them have potential to alter the GUS activity. Experimental evidences suggest that compounds in some dietary spices [8], D-glucaro-1,4-lactone in vegetables [9] had a decreasing role on the of $\beta$-glucuronidase activity. Besides, it was reported that activity of GUS enzyme extracted from the plant tissues was also influenced by interfering secondary plant compounds. Those secondary compounds were phenolic compounds, endogenous flavonols and proanthocyanidins and were reported as likely the inhibitor groups [10]. Thus it is possible that those plants may take a role in cancer prevention by their antioxidant and GUS inhibition capacities.

Pistacia terebinthus (Anacardiaceae) is one of the 20 Pistacia species widely distributed in the Mediterranean region and Asia. This plant has different biological activities and is used in different traditional cures such as treatment of burns, asthma and can be used as antiseptic for bronchitis in Turkey. Its fruit extracts were found to have flavonoids apigenin, luteolin, luteolin 7-O-glucoside, quercetin and kaempferol. Due to rich secondary compounds its fruits and resin were reported to have high antioxidant, antimicrobial, anti-inflammatory and cytotoxic properties [11]. But there wasn't any study indicating the capacity of GUS inhibition for any part of $P$. terebinthus and antioxidant capacity of its leaves. The aim of this study was to investigate inhibitory potential of $P$. terebinthus (from the Karaburun Region Turkey) leave extracts on E. coli glucuronidase and to investigate their bioactivities. Besides, bioactive compounds in leave extract were characterized by high performance liquid chromatography (HPLC) which were responsible for GUS inhibition.

\section{Experimental}

\subsection{Materials}

Fresh $P$. terebinthus leaves were collected in June from Karaburun region in Turkey. $\beta$-Glucuronidase (from $E$. coli), 4-methylumbelliferone (4-MU; sodium salt), 4-methylumbelliferone$\beta$-D-glucuronide (4-MUG), phenolphthalein glucuronide, 2,2'azinobis 3-ethylbenzothiazoline-6-sulfonic acid diammonium salt (ABTS), Nutrient broth were purchased from Sigma (Steinheim, Germany). Trolox (6-hydroxy-2,5,7,8,-tetramethylchroman2-carboxylic acid), and potassium persulfate $\left(\mathrm{K}_{2} \mathrm{O}_{8} \mathrm{H}_{8}\right)$ from Fluka (Steinheim, Germany). Dimethylsulphoxide (DMSO) was purchased from Carlo Erba (Spain). Stock cultures of E. coli (ATTC 8739), Staphylococcus aureus ATCC 29213 (Agricultural Research Service Culture Collection, IL, USA) were kindly provided by Ege University, Biology Department. All other reagents were of analytical and HPLC grade.

\subsection{Preparation of crude extracts from P. terebinthus leaves}

Collected leaves were washed with deionized water and dried at $37^{\circ} \mathrm{C}$ for 3 days. Dried leaves were powdered and $5 \mathrm{~g}$ of it was extracted with $100 \mathrm{ml}$ of $80 \%$ ethanol-water solution for $2 \mathrm{~h}$ at $37^{\circ} \mathrm{C}$. Then, ethanol was removed with rotary evaporator and final dry extract was obtained after freeze drying of liquid extract.

\subsection{HPLC characterization of crude extract}

In this study, HPLC analysis given in literature was used to identify flavonoids and other phenolic compounds [12]. For this purpose, the crude extract was dissolved in DMSO with a concentration of $5 \mathrm{~g}^{-1}$ and was injected to the HPLC. The HPLC equipment used was a Hewlett-Packard series HP 1100 equipped with a diode array detector. The stationary phase was a C18 LiChrospher 100 analytical column $(250 \mathrm{~mm} \times 4 \mathrm{~mm}$ i.d. $)$ with a particle size of $5 \mathrm{~mm}$ thermostated at $30^{\circ} \mathrm{C}$. The flow rate was $1 \mathrm{ml} \mathrm{min}^{-1}$ and the absorbance changes were monitored at $280 \mathrm{~nm}$ for the detection of simple phenolic compounds and flavonoids, at $368 \mathrm{~nm}$ for the detection of quercetin and kaempferol.

\subsection{Determination of antioxidant activity}

The trolox equivalent antioxidant capacity (TEAC) assessment was performed in terms of radical scavenging ability according to the ABTS $/ \mathrm{K}_{2} \mathrm{~S}_{8} \mathrm{O}_{2}$ method [13]. Serial dilutions of plant extract were prepared and scavenging ability of samples for ABTS radical cation compared with trolox standard absorbance curve. The antioxidant activity results were reported as the trolox equivalent antioxidant capacity. The values of TEAC were given as mmol trolox/g of $P$. therebinthus leaf extract and comparison was made with other synthetic antioxidants; Butylated hydroxyanisole (BHA), Butylated hydroxytoluene (BHT) and natural antioxidant; catechin and ascorbic acid.

\subsection{Determination of the MIC Values by micro-dilution assay}

In the present study, a serial 2-fold micro-broth dilution method [14] was performed to determine the MIC of plant extract on two GUS producing bacteria of E. coli; gram(-) and S. aureus; gram(+). Firstly the crude extract of $P$. terebinthus was dissolved in DMSO for a concentration of $100 \mathrm{mg} \mathrm{ml}^{-1}$ as stock solution. Two fold serial dilutions were carried out using sterile deionized water. Then $100 \mu \mathrm{L}$ of extracts at different concentrations and $95 \mu \mathrm{l}$ nutrient broth were added in each well of 96 well microplate. Each well was inoculated with $5 \mu \mathrm{l}$ of $6 \mathrm{~h}$ incubated bacterial suspensions after being standardized by adjusting their optical densities at $420 \mathrm{~nm}$ by UV spectrophotometer (PerkinElmer). Negative controls were performed for each strain by serial dilutions of DMSO. The assay plates were incubated at $37^{\circ} \mathrm{C}$ for $24 \mathrm{~h}$ and the growth kinetic assays for each strain were performed duplicate by growth curves and observed as turbidity determined by a microplate reader (Varioskan Flash, Thermo) at $620 \mathrm{~nm}$. MIC results for extracts were reported as $\mathrm{mg} \mathrm{ml}^{-1}$.

\subsection{Dose-response analysis}

To assess the inhibitory effect of plant extract doses on glucuronidase activity and to find $\mathrm{IC}_{50}$ values, $1-5 \mu \mathrm{g}$ extracts were previously incubated with GUS at $37^{\circ} \mathrm{C}$ for $30 \mathrm{~min}$ in $600 \mu \mathrm{l}$ phosphate buffer at $\mathrm{pH} 6.8$ in 24-well plate. Measurements of GUS activity were performed using the substrate 4-methylumbelliferyl$\beta$-D-glucuronide (4-MUG) where final concentrations of extracts in reaction mixture were in range of 1.67 and $8.33 \mu \mathrm{g} \mathrm{ml}^{-1}$. The activity was measured as the rate of production of fluorescent methylumbelliferone (4-MU), resulting from the hydrolysis of the substrate 4-methyllumbellyferone glucuronide by microplate reader (Varioskan Flash, Thermo) at excitation and emission wavelengths of 360 and $450 \mathrm{~nm}$, respectively [15]. Activity was expressed as the production rate of $1 \mu \mathrm{M}$ 4-methylumbelliferone (4-MU) liberated per minute for mg enzyme and it was determined by standard curve obtained by plotting 4-MU as standard. Assay was performed in duplicate and dose response data was normalized by percentage taking control as $100 \%$.

\subsection{Kinetic analysis}

To determine kinetic parameters, activity measurements were performed according to the given procedure by Fishman and Bernfeld [16] with slight modifications. In the absence and presence of 

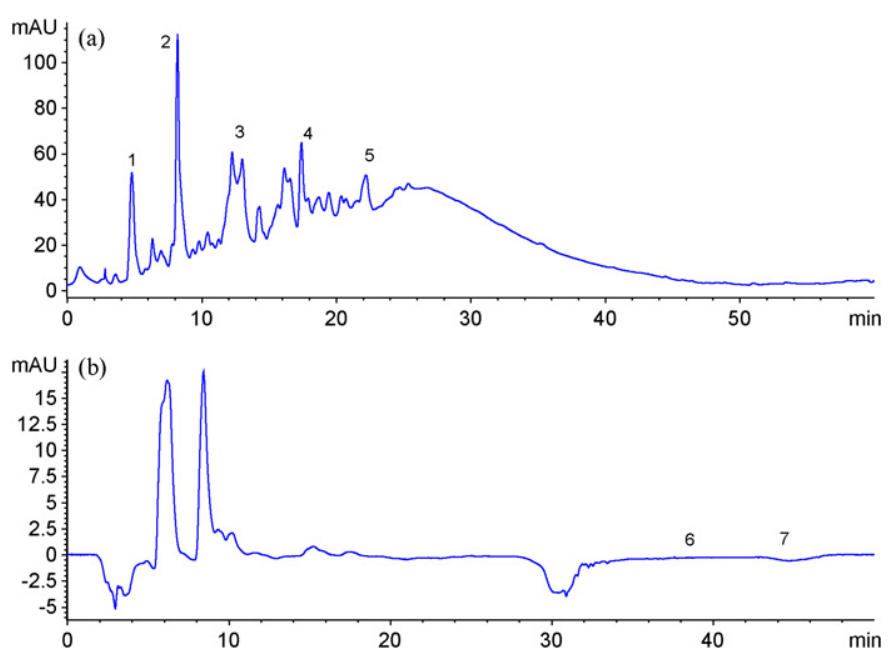

Fig. 1. HPLC chromatogram of $P$. terebinthus crude extract (a) at $280 \mathrm{~nm}-1$ : catechin; 2: unknown; 3: luteolin-7-glycoside; 4: apigenin-7-glycoside; 5: luteolin. (b) At $368 \mathrm{~nm}-6$ and 7 show the retention times of the standards, quercetin and kaempferol, respectively and there was not any peak obtained belonging to these flavonoids.

Table 1

Comparison of $P$. terebinthus crude extract antioxidant activity with natural and synthetic antioxidants.

\begin{tabular}{lc}
\hline Samples & $\begin{array}{l}\text { TEAC values } \\
\text { (mmol trolox/g antioxidant) }\end{array}$ \\
\hline (+)-catechin & 8.01 \\
BHA & 6.66 \\
BHT & 0.59 \\
Ascorbic acid & 6.45 \\
Crude extract of $P$. terebinthus leaf & 85.06 \\
\hline
\end{tabular}

plant extracts at concentrations of 1.39 and $2.08 \mu \mathrm{g} \mathrm{ml}^{-1}$, GUS was incubated with substrate phenolphthalein- $\beta$-D-glucuronide with varying concentrations of $125-2000 \mathrm{nM}$ for $30 \mathrm{~min}$. After $30 \mathrm{~min}$ reaction was terminated by the addition of glycine buffer $(\mathrm{pH}$ 10.4). Assay was performed in duplicate and the absorbance values of phenolphthalein formed was measured spectrophotometrically at $551 \mathrm{~nm}$ using Varioskan Flash (Thermo). Results were; $V$ was the rate of reaction, $V_{\max }$ was the maximum velocity, $K_{\mathrm{m}}$ is the Michaelis constant, and [S] was the substrate concentration.

\subsection{Calculations}

For the inhibition and kinetic data evaluation, GraphPad Prism 5.0 software was used. The kinetic parameters $\left(K_{\mathrm{m}}, V_{\max }\right.$ and $\left.K_{i}\right)$ were calculated by nonlinear curve fitting in the activity versus substrate concentration diagram, using the mixed type inhibition

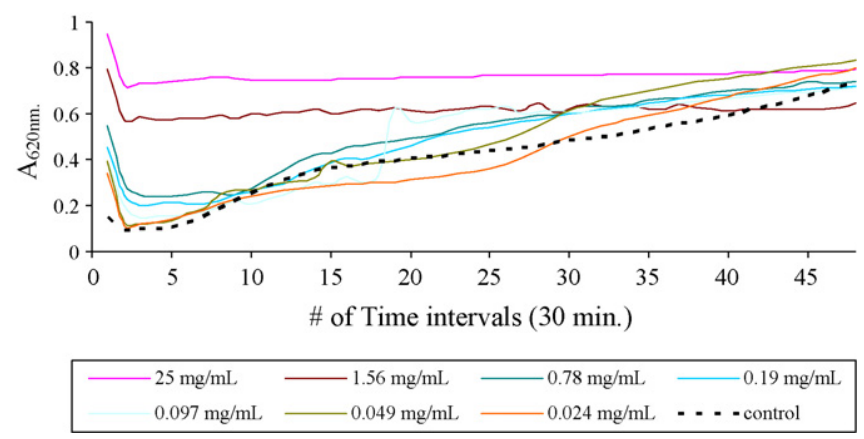

Fig. 2. Effects of varying concentrations of crude extract of $P$. terebinthus leaf on gram positive bacteria: S. aureus. Dashed line indicates the control growth.

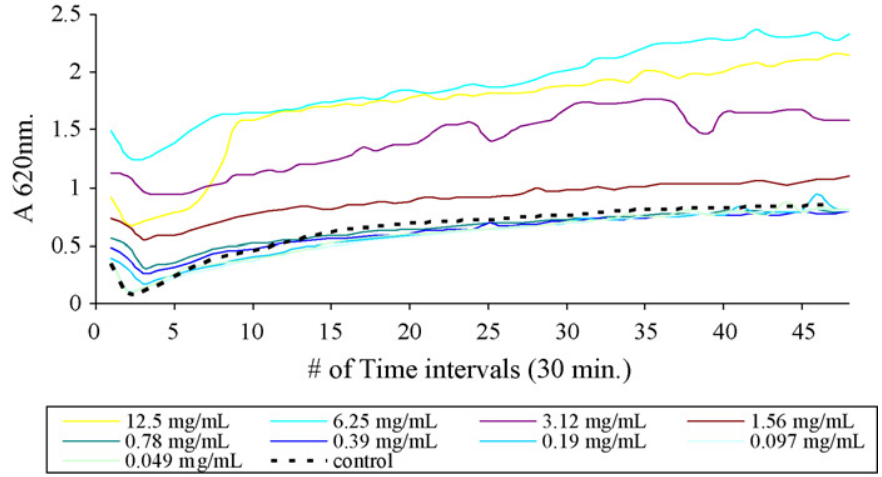

Fig. 3. Effects of varying concentrations of crude extract of $P$. terebinthus leaf on gram positive bacteria: E. coli. Dashed line indicates the control growth.

model, which was derived the Michaelis-Menten approach. In this model given below, alpha $(\alpha)$ is one of the important parameter affecting the mechanism. At very high $\alpha$ values that is much greater than 1 , mechanism is explained by competitive inhibition and very low $\alpha$ values ( $\alpha$ «1) indicate uncompetitive inhibition. If it is around $1(\alpha<1$ or $\alpha>1)$ at a certain value, mechanism can be described as mixed type inhibition which explains the deviation from noncompetitive and competitive nature [17]. If it is equal to 1 , noncompetitive inhibition takes place.

$v=\frac{V_{\max }[\mathrm{S}]}{K_{\mathrm{m}}\left(1+[\mathrm{I}] / K_{i}\right)+[\mathrm{S}]\left(1+[\mathrm{I}] / \alpha K_{i}\right)} \quad$ (mixed type inhibition)

The $\mathrm{IC}_{50}$ values were calculated by fitting a sigmoidal dose-response curve. The quantified results were given as means \pm S.D. (standard deviations) or \pm S.E. (standard errors of parameter estimates) and were compared using ANOVA with Dunnett's multiple comparison test. Differences with $p<0.05$ were considered significant.

\section{Results and discussion}

\subsection{HPLC characterization}

In the present study, the extract prepared from $P$. terebinthus leaves collected from Karaburun region was investigated for their flavonoids and phenolic compounds. The flavonoid compounds, luteolin, luteolin-7-glycoside and apigenin-7-glycoside and phenolic compounds such as catechin were detected in $P$. terebinthus

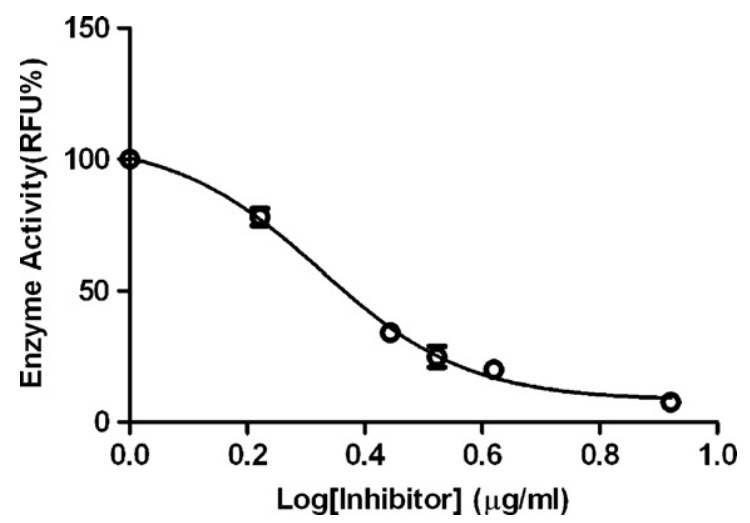

Fig. 4. Dose response curves for GUS activity and varying concentrations of $P$. terebinthus leaf crude extract: $0,1.67,2.78,3.33,4.17,8.33\left(\mu \mathrm{g} \mathrm{ml} l^{-1}\right.$ reaction mixture). Activity values ( $\mu \mathrm{M} 4-\mathrm{MU} \mathrm{min}^{-1} \mathrm{mg}^{-1}$; data not shown) corresponding to each concentration of extract were normalized according to control sample's activity considering as $100 \%$. IC 50 value as $2.1 \pm 0.2 \mu \mathrm{g} \mathrm{ml}^{-1}\left(\log \mathrm{IC}_{50}: 0.3\right)$ were obtained by curve fitting using GraphPad Prism 5.0. 

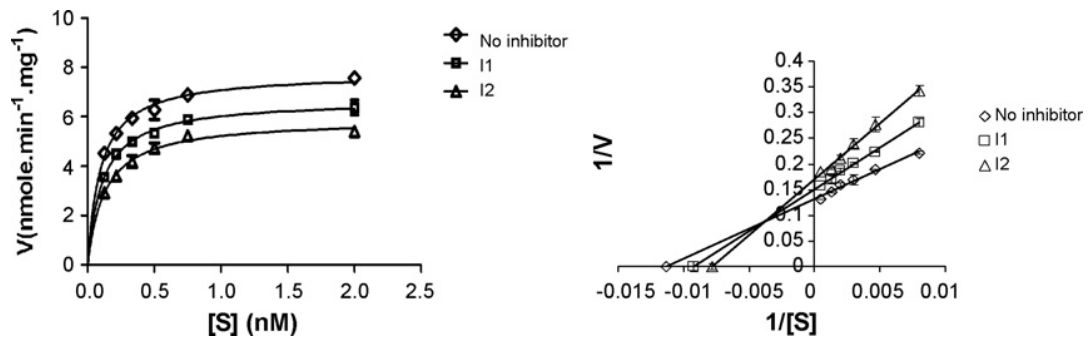

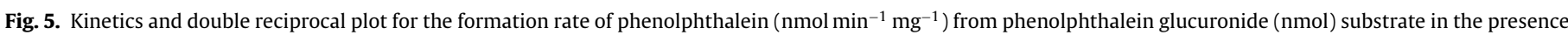
or absence of inhibitor. Inhibitor concentrations were approximately $0,1.39$ and $2.08 \mu \mathrm{g} \mathrm{ml}^{-1}$. Each point represents average of duplicate incubations.

crude leaf extract at $280 \mathrm{~nm}$ (Fig. 1a). On the other hand, the flavonoid compounds, quercetin and kaempferol were not detected in crude leaf extract at $368 \mathrm{~nm}$ (Fig. 1b). Those secondary compounds detected by HPLC may play an important role on enzyme activities. Luteolin-7-glycoside and its aglycon, luteolin, were potent inhibitors of DNA topoisomerase I by binding of them to the enzyme $[18,19]$. The pharmacological inhibition of this enzyme was important since topoisomerase inhibitors have emerged as anticancer, anti-microbial and anti-parasitic agents. In another study, Tadera et al. [20] investigated the inhibition effect of flavonoids on $\alpha$-glucosidase and they concluded that luteolin was potent inhibitor of it because of its $-\mathrm{OH}$ group and the hydroxyl substitution on the ring.

\subsection{Antioxidant capacity of crude extract of P. terebinthus leaf}

The antioxidant activities of ascorbic acid, synthetic antioxidants and crude extract of $P$. terebinthus leaf were given in Table 1 . The extract obtained from the $P$. terebinthus leaf has considerably higher antioxidant activity when compared with the other synthetic and natural antioxidants; approximately 12-fold higher antioxidant capacity than those of BHA and ascorbic acid. This may probably due to the high flavonoid and phenolic compounds content of crude extract since the extract also exhibits 10 -fold higher antioxidant activity than (+)-catechin. These results indicated that high antioxidant activity of plant extract may take an effective role in cancer prevention by protecting cells from the damage of free radicals.

\subsection{Antimicrobial activity of crude extract of P. terebinthus leaf}

P. terebinthus leaf extract showed antimicrobial activity against $S$. aureus with a minimum inhibition concentration of $\leq 1.56 \mathrm{mg} \mathrm{ml}^{-1}$ (Fig. 2). On the other hand, there was no antimicrobial effect of crude extract on E. coli (Fig. 3). Since E. coli is gram negative bacteria and has a lipopolysaccharide outer membrane, transfer of molecules is achieved through the cell membrane with porins. Antimicrobial resistance in gram (-) organism is frequently mediated via alterations in an outer membrane porin, which significantly decreases the ability of antimicrobial to penetrate the outer plasma membrane and reach its site of action [14]. Thus ability of molecules to pass through these channels was influenced by their size and shape. Flavonoids found in leaf extract probably could not pass through the cell membrane.

\subsection{Effect of crude extract concentration on GUS activity}

The concentrations of extracts that inhibit the hydrolysis of substrates by $50 \%$ ( $\mathrm{IC}_{50}$ values) were determined fluorometrically by increasing concentrations of test compounds on enzyme activity. Results showed that plant extracts provided high inhibition capacity and under the present study conditions. At $8.3 \mu \mathrm{g} \mathrm{ml}^{-1}$ extract concentration, enzyme was not completely inhibited but the highest inhibition was achieved where GUS enzyme was inhibited approximately $92.4 \%$ (activity decreased from $556.7 \pm 30.8$ to $42.6 \pm 7.4 \mu \mathrm{M} 4$-MUG $\mathrm{min}^{-1} \mathrm{mg}^{-1}$, data was not shown). According to dose response curves (Fig. 4 ), $\mathrm{IC}_{50}$ value was calculated as $2.113 \pm 0.2 \mu \mathrm{g} \mathrm{ml}^{-1}\left(\log \mathrm{IC}_{50}: 0.325\right)$. Those high inhibition capacities of different doses extracts were possibly due to those secondary compounds characterized by HPLC. Some compounds of plant origin were reported as the potent GUS inhibitor [9] and phenolic compounds were reported as one likely inhibitor group [21,10]

\subsection{Kinetic analysis}

The results of the kinetic analysis of inhibition at varying concentrations of $P$. terebinthus leave extract were analyzed by nonlinear regression curve fitting for mixed type inhibition model derived from Michaelis-Menten kinetics. Results of velocity versus substrate concentrations were given in Fig. 5 and each point represented the average of duplicate incubations. Inhibitor effects were compared to control and statistically considered as significant $(p<0.05)$. $V_{\max }$ and $K_{\mathrm{m}}$ values were determined as $7.81 \pm 0.16 \mathrm{nmol} \mathrm{min}^{-1} \mathrm{mg}^{-1}$ and $96.94 \pm 9.68 \mathrm{nM}$. Inhibition constant $\left(\alpha K_{i}\right)$ for mixed type inhibitors was also found as $7.00 \pm 0.93 \mathrm{nM}$ having $\alpha$ value $2.17 \pm 0.93$. Inhibition mechanism was also supported by the Lineweaver-Burk linear transformations and the resulting double reciprocal plot indicated that common intersect point of straight lines was at the II quadrant (Fig. 5).

\section{Conclusions}

According to the results, it was found that $P$. terebinthus had a high antioxidant capacity indicating a possible preventive role in cancer risks by eliminating the free radicals attack. Two GUS producing bacteria were used to determine the antimicrobial activity of $P$. terebinthus, and it was found that although plant extract showed an antimicrobial effect on gram positive $S$. aureus, it was ineffective on gram negative $E$. coli. Kinetic studies indicated an effective inhibition of GUS enzyme. Thus, this effective inhibition might take a preventive role for deglucuronidation in human intestine caused by microbial flora. When the crude extract of $P$. terebinthus was characterized to have an idea about possible inhibitor compounds exist in its nature, HPLC characterization of the extract indicated that this plant was rich in secondary plant compounds such as luteolin and luteolin-7-glucoside. It was found that these secondary metabolites in crude extract exhibited high antioxidant activity and in literature most of them were reported to have anticarcinogenic property. This antioxidant property together with the effective GUS inhibition makes crude extract an alternative for phytopharmacy. In conclusion, this study showed that the use of $P$. terebinthus extract inhibited $E$. coli GUS activity effectively and thus it might reduce potential cancer risks. Future studies should be performed in order to investigate the inhibitory effect of secondary compounds in P. terebinthus on GUS activity separately. 


\section{References}

[1] D. Beaud, P. Tailliez, J. Anba-Mondoloni, Microbiology 151 (2005) 2323-2330

[2] R. Chouiter, I. Roy, C. Bucke, J. Mol. Catal. B: Enzym. 50 (2008) 114-120.

[3] J.W. Leung, Y. Liu, P.S.C. Leung, R.C.Y. Chan, J.F. Inciardi, A.F. Cheng, Gastrointest. Endosc. 54 (2001) 346-350.

[4] M.E. Cardona, E. Norin, T. Midvedt, Microb. Ecol. Health Dis. 18 (2006) 38-41.

[5] N. Nalini, K. Sabitha, P. Viswanathan, V.P. Menon, J. Ethnopharmacol. 62 (1998) $15-24$.

[6] J. Rafter, M. Govers, P. Martel, D. Pannemans, B. Pool-Zobel, G. Rechkemmer, I. Rowland, S. Tuijtelaars, J. Loo, Eur. J. Nutr. 43 (2004) 1203-1206.

[7] T. Fujisawa, K. Aikawa, T. Takahashi, S. Yamai, K. Watanabe, Y. Kubota, M. Miyaoka, Lett. Appl. Microbiol. 32 (2001) 123-125.

[8] T. Devasena, V.P. Menon, Phytother. Res. 17 (2003) 1088-1091.

[9] J.W. Lampe, S.S. Li, J.D. Potter, I.B. King, J. Nutr. 132 (2002) 1341-1344.

[10] R. Serres, B. McCown, E. Zeldin, Plant Cell Rep. 16 (1997) 641-646.

[11] G. Topçu, M. Ay, A. Bilici, C. Sarıkürkçü, M. Öztürk, A. Ulubelen, Food Chem. 103 (2007) 816-822.
[12] E. Altıok, D. Bayçın, O. Bayraktar, S. Ülkü, Sep. Purif. Technol. 62 (2008) $342-348$

[13] E. Altık, S. Ülkü, O. Bayraktar, Food Sci. Technol. Res. 13 (2007) 321-326.

[14] D. Kaçar, Screening of some plant species for their total antioxidant and antimicrobial activities, M.S. Thesis, Izmir Institute of Technology, Department of Biotechnology and Bioengineering, Izmir, 2008, p. 54.

[15] J.H. Glaser, H.E. Conrad, J. Biol. Chem. 254 (1979) 6588-6597.

[16] W.H. Fishman, P. Bernfeld, Methods Enzymol. 1 (1955) 262-269.

[17] R.A. Copeland, Enzymes: A Practical Introduction to Structure, Mechanism and Data Analysis, second ed., Wiley, New York, 2000.

[18] M. Galvez, C.M. Cordero, M.L. Lazaro, F. Cortes, M.J. Ayusa, J. Ethnopharmacol. 88 (2003) 125-130.

[19] A.R. Chowdhury, S. Sharma, S. Mandal, A. Goswami, S. Mukhopadhyay, K.M. Majumder, Biochem. J. 366 (2002) 653-661.

[20] K. Tadera, Y. Minami, K. Takamatsu, T. Matsouka, J. Nutr. Sci. Vitaminol. 52 (2006) 149-153.

[21] K. Revesz, A. Tütto, E. Margittai, G. B'anhegyi, J.E. Magyara, J. Mandla, M. Csala, Int. J. Biochem. Cell B 39 (2007) 922-930. 